\title{
Self-regulation following prostatectomy: Phase-specific self-efficacy beliefs for pelvic-floor exercise
}

\author{
S. Burkert ${ }^{\prime *}$, N. Knoll', U. Scholz ${ }^{2}$, J. Roigas ${ }^{3}$ and O. Gralla ${ }^{4}$ \\ 'Institute of Medical Psychology, Charité Universitätsmedizin Berlin, Germany \\ ${ }^{2}$ Department of Psychology, University of Bern, Switzerland \\ ${ }^{3}$ Vivantes Klinikum am Urban, Berlin, Germany \\ ${ }^{4}$ Urologie am Ring, Cologne, Germany
}

Objective. Beliefs in one's ability to perform a task or behaviour successfully are described as self-efficacy beliefs (Bandura, 1977). Since individuals have to deal with differing demands during a behaviour-change process, they form phase-specific selfefficacy beliefs directed at these respective challenges. The present study, based on the Health Action Process Approach (Schwarzer, 200I), examines the theoretical differentiation, relative importance, and differential effects of four phase-specific selfefficacy beliefs, including task self-efficacy, preactional self-efficacy, maintenance selfefficacy, and recovery self-efficacy.

Design. In a prospective longitudinal study, 112 prostatectomy-patients received questionnaires at 2 days, 2 weeks, 1 month, and 6 months post-surgery.

Methods. Participants provided data on phase-specific self-efficacies as well as phase indicators of health-behaviour change, that is, intentions, planning, and pelvic-floor exercise. Hierarchical regression analyses were conducted to test the study hypotheses.

Results. Task self-efficacy was not uniquely associated with intentions. Preactional self-efficacy was related to action planning. Maintenance self-efficacy did not predict behaviour. Recovery self-efficacy was associated with re-uptake of pelvic-floor exercise after relapses only.

Conclusion. Findings underline the importance of differentiating between task selfefficacy and preactional self-efficacy during early phases of behaviour change as well as of considering the occurrence of relapses as a moderator of potential effects of recovery self-efficacy on the maintenance of behaviour change. Advanced knowledge on distinct, phase-specific self-efficacy beliefs may facilitate the design of effective tailored interventions for behaviour change.

\footnotetext{
* Correspondence should be addressed to Silke Burkert, PhD, Charité - Universitätsmedizin Berlin, Institute of Medical Psychology, Luisenstr. 7, 10117 Berlin, Germany (e-mail: silke.burkert@charite.de).
} 
Self-efficacy beliefs based on Bandura's (1997) social cognitive theory refer to selfconfidence in one's own capacity to prepare, initiate, and maintain behaviour, even if obstacles emerge. Self-efficacy beliefs depend on the context and on the level of perceived difficulty of the behaviour (Bandura, 1997). For example, during the process of health-behaviour change, individuals face different tasks with individually different levels of difficulty: To pass through the health-behaviour change process, individuals first have to form intentions. Afterwards, they have to plan, initiate, and maintain the behaviour, and sometimes recover from relapses (Schwarzer, 2001). Because each phase provides its own specific challenges, phase-specific self-efficacy beliefs specifically concern challenges in respective phases (Marlatt, Baer, \& Quigley, 1995). The aim of the present paper was to enhance the existing knowledge on phase-specific self-efficacies (Scholz, Sniehotta, \& Schwarzer, 2005; Schwarzer, 2008) by differentiating and testing the predictive validity of four phase-specific self-efficacies in health-behaviour change.

\section{Phase-specific self-efficacy beliefs}

Beliefs in one's ability to perform a task or behaviour successfully are described as selfefficacy beliefs (Bandura, 1977). Originally, self-efficacy was conceptualized as domainor action-specific. Later, self-efficacy was related to specific phases in health-behaviour change (Marlatt et al., 1995; Rodgers, Hall, Blanchard, McAuley, \& Munroe, 2002). The Health Action Process Approach (HAPA; Schwarzer, 2001) is a model for the investigation of health-behaviour change that places a special emphasis on self-efficacy beliefs, with self-efficacy being an important predictor in all phases of change (Abraham, 2008; Lippke, Ziegelmann, \& Schwarzer, 2005; Renner, Spivak, Kwon, \& Schwarzer, 2007). The model distinguishes between a preintentional, a preactional phase, and an action phase (Lippke et al., 2005; Scholz et al., 2005; Wiedemann et al., 2009). Phase-specific self-efficacy beliefs are differentiated and supposed to operate differently in these phases of change (Schwarzer, 2001). Previous research mainly focused on three phase-specific self-efficacies (i.e., task, maintenance, and recovery self-efficacy; Schwarzer, 2008). The present study aims at differentiating between four phase-specific self-efficacy beliefs. Specifically, we investigate preactional self-efficacy and newly test its phase-specific predictive validity against preintentional task self-efficacy, maintenance self-efficacy, and recovery self-efficacy. Self-efficacy beliefs are introduced in order of theoretical appearance below.

(1) Task self-efficacy is located in the preintentional phase which comprises persons who are not intending to change their behaviour. If individuals believe to be vulnerable to health threats (risk perception) and expect that the uptake of a specific health behaviour has more advantages than disadvantages (outcome-expectancies), they are more likely to form an intention to exercise regularly. Task self-efficacy refers to individuals' overall confidence in their ability to enact a behaviour. High task self-efficacy increases the probability of forming an intention to change behaviour (Renner et al., 2007; Rodgers et al., 2002; Schwarzer et al., 2007).

In the preactional phase, (2) preactional self-efficacy helps to pass from intentions to action, when behaviour change is planned and prepared. Planning helps to initiate health behaviour (Leventhal, Singer, \& Jones, 1965). In action plans, behaviour is linked to situational cues in pre-deciding when, where, and how to perform the new behaviour (Gollwitzer, 1999; Gollwitzer \& Sheeran, 2006). Thus, action planning can be considered as a last preparatory step before action initiation. Action preparation and implementation is supported by preactional self-efficacy, which refers to the confidence 
in one's ability to prepare and start a new behaviour even if its implementation has to be prepared thoroughly first. Task self-efficacy and preactional self-efficacy have not yet been operationalized as distinct constructs, and preactional self-efficacy has rarely been investigated to date (Chow \& Mullan, 2010; Luszczynska \& Schwarzer, 2003).

In the action phase of the HAPA, behaviour is protected against or recovered in case of relapses. Evidence emerged that self-efficacy in the preintentional and preactional phase can be distinguished from self-efficacy during the action phase (Luszczynska \& Schwarzer, 2003). In the action phase, two further self-efficacy beliefs may help to maintain health behaviour. (3) Maintenance self-efficacy refers to beliefs in one's ability to cope with obstacles that may interfere with the maintenance of health behaviour, and is therefore relevant when behaviour is already initiated or enacted (Scholz et al., 2005).

(4) Recovery self-efficacy is needed to return to the intended behaviour after a relapse. There are consistent findings that maintenance and recovery self-efficacy are crucial to sustain or return to regular performance of various health behaviours (Rodgers $e t$ al., 2002; Scholz et al., 2005; Schwarzer et al., 2007).

To sum up, phase-specific self-efficacy beliefs should show different predictive patterns with regard to different phase-specific outcomes of behaviour change, including intentions, planning, and health-behaviour performance. In the present study, phasespecific self-efficacy beliefs are investigated in the context of health-behaviour change following radical prostatectomy.

\section{Pelvic-floor exercise in prostatectomy patients}

Prostate cancer is one of the most prevalent cancers in men (Ferlay, Bray, Pisani, \& Parkin, 2001). One treatment for localized prostate cancer is radical prostatectomy (RPE; Debruyne \& Beerlage, 2000), where the entire prostate is surgically removed. Urinary incontinence as a common post-operative comorbidity sets in immediately postsurgery and recedes within the first 12 months in most patients (Basillote, Ahlering, Skarecky, Lee, \& Clayman, 2004). To control post-operative incontinence, patients are recommended to implement pelvic-floor exercise (Hunter, Moore, \& Glazener, 2007). There are no general recommendations concerning the training frequency and intensity, but the outer pelvic-floor muscles can be strengthened by several exercise units each day. Patients are encouraged to continue exercising at least as long as incontinence persists (Dorey, Glazener, Buckley, Cochran, \& Moore, 2009).

\section{Aims and hypothesis}

To our knowledge, previous studies based on the HAPA only investigated three phasespecific beliefs empirically, and did not differentiate between task and preactional selfefficacy (Luszczynska \& Schwarzer, 2003; Renner et al., 2007; Scholz et al., 2005). In this study, we focused on testing the relative impact and unique predictive validity of four phase-specific self-efficacies on phase-specific outcomes, namely on intentions (preintentional phase), planning processes (preactional phase), and behaviour maintenance including recovery (actional phase). A study design was chosen in which these phase-specific outcomes were assessed at three measurement points after baseline, using two different measurement lags. That is, assessments and time lags in between were meant to parallel theoretically assumed milestones of behaviour change following surgery. In particular, we tested the following hypotheses: 
(1) Task self-efficacy (as compared to preactional, maintenance, and recovery selfefficacy) is the strongest predictor for intentions 2 weeks post-surgery.

(2) Preactional self-efficacy (as compared to task, maintenance, and recovery selfefficacy) is the strongest predictor for action planning 2 weeks post-surgery.

(3) Maintenance self-efficacy (as compared to task, preactional, and recovery selfefficacy) is the strongest predictor for pelvic-floor exercise 1 month post-surgery.

(4) Recovery self-efficacy (as compared to task, preactional, and maintenance selfefficacy) is the strongest predictor for pelvic-floor exercise 6 months post-surgery, when actors are more likely to have experienced lapses in behaviour change already.

To further qualify the main effects in terms of phase-specificity, we explored whether the associations between phase-specific self-efficacy beliefs and phase-specific outcomes were moderated by phase indicators (i.e., intention strength, past behaviour, and relapses). For example, high intentions to change one's behaviour may indicate being in the preactional phase. Accordingly, especially individuals with high intentions and high preactional self-efficacy beliefs should report high levels of planning. Past behaviour can be used as an indicator of being in the maintenance phase. Maintenance self-efficacy should be associated more strongly with future behaviour in individuals, who were already active in the past. Lapsing into non-behaviour may indicate being in the recovery phase. Only individuals with setbacks should benefit from their recovery self-efficacy beliefs, as individuals who did not relapse are not in need of a high recovery self-efficacy to return to prior activity levels.

\section{Method}

\section{Design and sample}

Data were assessed from $N=112$ in patients of the Department of Urology at a German University Medical Centre. Patients were part of a couple study on adaptation to radical prostatectomy, were all in stable relationships and had comprehensive knowledge of the German language. All patients gave informed consent to participation in the study. Data were assessed using questionnaires at four waves, which were chosen to represent the different phases of the behaviour-change process.

Baseline measurements ( $t 1)$ took place in the hospital 2 days post-surgery when participants had received information about pelvic-floor exercise by physiotherapists. Information about and dealing with pelvic-floor exercise is assumed to foster intention formation, thus at $t 1$ patients were assumed to be in the preintentional phase. Patients were recommended not to start exercising as long as they were catheterized. Thus, in the days after surgery and discharge, patients were assumed to prepare for healthbehaviour change: They were assumed to be in the preactional phase 2 weeks postsurgery (t2), and to plan how to implement pelvic-floor exercise in their everyday life. One month post-surgery ( $t 3)$, participants were assumed to have already initiated exercising, that is, they were in the action phase of behaviour change, focussing on behaviour maintenance. Since maintenance over 6 months is difficult and highly prone to relapses, the 6 months post-surgery assessment $(\mathrm{t} 4)$ represented the recovery phase. Participants were differentiated in maintainers $(n=71,63.4 \%)$ and those who recovered after setbacks ( $n=41,36.6 \%$ ).

To test the relative importance of phase-specific self-efficacy beliefs, most were assessed at all times. However, we refrained from assessing preactional self-efficacy 
at 6 months post-surgery ( $t 4)$ as patients were expected to have completed the planning phase at this point. Other social-cognitive variables, pelvic-floor exercise, and further indicators of recovery (i.e:, catheterization, rehabilitation, and incontinence) were assessed at time points indicated in the section on measures.

Follow-up measurements via mail were completed by 106 individuals at 2 weeks (t2), by 100 individuals at 1 month ( $\mathrm{t} 3$ ), and by 94 individuals at 6 months post-surgery ( 4 ), resulting in a total dropout rate of $16 \%$. An institutional review board approved study procedures.

Participants' mean age was 62.8 years $(S D=6.0$ ). Patients were married ( $n=102$; $91.1 \%)$ or cohabiting $(n=10 ; 8.9 \%)$. Only $n=6(5.4 \%)$ were divorced and $n=6$ $(5.4 \%)$ were widowed. The majority of the patients $(n=99 ; 88.4 \%)$ had children. About half of the participants reported less than 12 years of schooling $(n=42 ; 37.5 \%)$, the other half $(n=63 ; 56.2 \%$ ) had 12 or 13 years of schooling. Seven participants did not provide data on their education. Most participants were retired ( $n=61 ; 54.5 \%$ ), the remainder were employed $(n=39 ; 34.8 \%)$ or unemployed $(n=7 ; 6.3 \%)$. Radical prostatectomy is recommended if the cancer has not metastasized (M0) or spread to lymph nodes (NO). Few patients' tumours ( $n=3 ; 2.7 \%)$ were classified as not palpable (T1NOM0), most patients' tumours were either palpable ( $n=85 ; 75.9 \%$; T2NOM0) or spread through the prostatic capsule ( $n=22,19.6 \%$; T3NOMO). All patients underwent radical prostatectomy, and $n=45(40.9 \%)$ of them received nerve-sparing surgery.

\section{Measures}

Means, standard deviations, Cronbach's alphas, and intercorrelations of the central variables are reported in Table 1.

Task self-efficacy was assessed by three items (e.g., 'I am confident that I can perform pelvic-floor exercise several times a week'; adapted from Scholz et al., 2005). Unless noted otherwise, response options for the social-cognitive variables ranged from 1 (does not apply at all) to 4 (applies exactly).

Preactional self-efficacy was measured with the item stem 'I am confident to start pelvic-floor exercise ...' and three different steps to action, that is, 'even if I have to learn these exercise techniques first', 'even if I have to force myself, to start immediately', and 'even if I have to plan how to implement pelvic-floor exercise in everyday life'.

Maintenance self-efficacy was measured by the item stem 'I am confident to perform pelvic-floor exercise regularly on a long-term basis ...' which was followed by five typical barriers that may hamper behavioural maintenance, for example, 'even if I cannot see any positive changes concerning the incontinence immediately' (adapted from Scholz et al., 2005).

Recovery self-efficacy was measured by the stem 'I am confident that I can return to regular pelvic-floor exercise ...' was followed by three items (e.g., 'even if I have relapsed several times'; adapted from Scholz et al, 2005).

Except for preactional self-efficacy (not assessed at $\mathrm{t} 4$ ), all phase-specific self-efficacy beliefs were assessed at all measurement points in time. Principal components analyses (PCA) with varimax rotation of the 14 self-efficacy items were computed at the four measurement points, extraction criteria were Eigenvalue $>1$ or the elbow criterion (Tabachnick \& Fidell, 2001). PCAs yielded distinct components with three items for task self-efficacy, three for preactional self-efficacy, five for maintenance self-efficacy, and three for recovery self-efficacy. Looking at all three assessments, between $73 \%$ and $82 \%$ 
Table I. Central constructs: means (M), standard deviations (SD), internal consistencies (Cronbach's $\alpha$ ), and intercorrelations

\begin{tabular}{|c|c|c|c|c|c|c|c|c|c|c|c|c|c|c|c|c|c|c|c|c|c|c|c|c|c|}
\hline Construct & M & $S D$ & $\alpha$ & 2 & 3 & 4 & 5 & 6 & 7 & 8 & 9 & 10 & 11 & 12 & 13 & 14 & 15 & 16 & 17 & 18 & 19 & 20 & 21 & 22 & 23 \\
\hline I risk t I & 2.71 & 0.86 & .89 & -.20 & .28 & -.19 & .14 & -.07 & -.11 & -.16 & -.19 & -.16 & -.13 & -.23 & -.10 & -.19 & -.12 & -.15 & -.17 & .12 & -.07 & -.09 & -.03 & .08 & 18 \\
\hline 2 pout $\mathrm{tl}$ & 3.83 & 0.37 & .68 & & -.28 & .48 & .13 & .23 & .30 & .37 & .17 & .24 & .30 & .23 & .33 & .11 & .25 & .19 & .17 & -.06 & .01 & .10 & .09 & -.03 & -.15 \\
\hline nout $\mathrm{tl}$ & 1.86 & 0.72 & .50 & & & -.18 & .02 & .01 & -.11 & -.05 & -.19 & -.09 & -.12 & -.18 & -.07 & -.03 & -.14 & -.03 & -.11 & .08 & -.12 & -.16 & -.22 & -.02 & .15 \\
\hline tse tl & 3.67 & 0.49 & .85 & & & & .30 & .47 & .61 & .56 & .29 & .47 & .36 & .36 & .25 & .30 & .29 & .22 & .12 & -.04 & .14 & .17 & .15 & -.07 & .06 \\
\hline 5 pse tl & 3.38 & 0.73 & .86 & & & & & .42 & .41 & .23 & .17 & .14 & .31 & .26 & .04 & .16 & .30 & .07 & .08 & .01 & .18 & .11 & .03 & .03 & .00 \\
\hline 6 mse tl & 3.21 & 0.74 & .91 & & & & & & .49 & .36 & .24 & .24 & .20 & .28 & .14 & .15 & .19 & -.01 & .01 & .04 & .14 & .19 & .05 & -.01 & .06 \\
\hline 7 rse tl & 3.55 & 0.61 & .98 & & & & & & & .16 & .38 & .38 & .30 & .36 & .12 & .27 & .28 & .13 & .07 & .02 & .31 & .25 & .25 & .01 & .05 \\
\hline 8 int $\mathrm{tl}$ & 3.77 & 0.46 & .88 & & & & & & & & .14 & .41 & .24 & .22 & .22 & .37 & .25 & .11 & .12 & .17 & .22 & .12 & .08 & -.04 & .09 \\
\hline 9 ap tl & 2.41 & 1.12 & .92 & & & & & & & & & .11 & .11 & .20 & .21 & .06 & .22 & .21 & .10 & -.02 & .14 & .15 & .05 & -.03 & .02 \\
\hline 10 tse t2 & 3.64 & 0.50 & .80 & & & & & & & & & & .34 & .29 & .41 & .67 & .49 & .32 & .05 & .01 & .10 & .08 & .16 & -.05 & .02 \\
\hline 11 pse $t 2$ & 3.29 & 0.81 & .84 & & & & & & & & & & & .42 & .22 & .29 & .30 & .30 & .21 & .02 & -.01 & .11 & .13 & -.02 & -.11 \\
\hline 12 mse t2 & 3.27 & 0.64 & .86 & & & & & & & & & & & & .31 & .31 & .09 & -.01 & .11 & .17 & .07 & .17 & .12 & .03 & .05 \\
\hline 13 rse t2 & 3.49 & 0.65 & .90 & & & & & & & & & & & & & .33 & .23 & .15 & .05 & -.01 & .01 & .03 & .11 & -.09 & -.17 \\
\hline $14 \mathrm{int}$ t2 & 3.69 & 0.48 & .76 & & & & & & & & & & & & & & .27 & .12 & .15 & -.01 & .05 & -.03 & -.01 & -.02 & -.06 \\
\hline 15 ap t 2 & 3.33 & 0.86 & .85 & & & & & & & & & & & & & & & .33 & .17 & -.03 & .08 & .03 & .08 & .01 & -.03 \\
\hline 16 pfe t2 & 143.85 & 163.92 & - & & & & & & & & & & & & & & & & .32 & -.17 & .04 & -.04 & -.00 & .01 & -.11 \\
\hline 17 pfe t3 & 241.57 & 234.49 & - & & & & & & & & & & & & & & & & & .01 & -.02 & -.10 & -.27 & .10 & -.10 \\
\hline 18 iciq t3 & 13.90 & 4.76 & - & & & & & & & & & & & & & & & & & & .08 & .05 & .02 & .24 & .50 \\
\hline 19 tse $t 4$ & 3.24 & 0.78 & .90 & & & & & & & & & & & & & & & & & & & .57 & .50 & .37 & .16 \\
\hline $20 \mathrm{mse} t 4$ & 2.28 & 0.87 & .89 & & & & & & & & & & & & & & & & & & & & .45 & .04 & .08 \\
\hline 21 rse $t 4$ & 3.45 & 0.74 & .98 & & & & & & & & & & & & & & & & & & & & & .14 & .11 \\
\hline 22 pfe t 4 & 131.48 & 130.50 & - & & & & & & & & & & & & & & & & & & & & & & .31 \\
\hline 23 iciq $t 4$ & 8.02 & 5.36 & - & & & & & & & & & & & & & & & & & & & & & & \\
\hline
\end{tabular}

Note. Coefficients $>=|.18|$ significant at $p<.05$; risk $=$ risk perception; pout/nout $=$ positive/negative outcome expectancies; tse $=$ task self-efficacy; pse $=$ preactional self-efficacy; mse = maintenance self-efficacy; rse = recovery self-efficacy; int = intention; ap = action planning; pfe = pelvic-floor exercise; iciq = incontinence. $N=112$. 
of the total variance was accounted for. PCA thus confirmed discriminant validity of four phase-specific self-efficacy constructs.

Pelvic-floor exercise was assessed at all points in time. At $\mathrm{t} 1$, participants additionally indicated whether they had already heard of pelvic-floor exercise prior to surgery and whether they already exercised $(0=$ no, $1=$ yes). Pelvic-floor exercise in minutes per week was assessed by indicating how many days and how many times a day participants had engaged in pelvic-floor exercise in the past week. In addition, they indicated how long they had usually exercised per session (in minutes). Frequencies and average duration per unit were multiplied to obtain a weighted measure of pelvic-floor exercise duration.

Risk perception regarding the development of incontinence symptoms was measured at $\mathrm{t} 1$ with three items. The stem 'Compared to other people of your age and gender, how likely do you think it is ...' was followed by different risks such as '... you are incontinent for a long time?' (adapted from Renner et al., 2007 and Schwarzer, 2008). Response options ranged from 1 (extremely unlikely) to 5 (extremely likely).

Positive and negative outcome expectancies were measured at $\mathrm{t} 1$ with two items each. The stem 'If I perform pelvic-floor exercise regularly, ...' was followed by consequences such as ' $\ldots$ then I can do something about the incontinence' or '... then it reminds me of my disease' adapted from Scholz et al. (2005).

Behavioural intentions for regular pelvic-floor exercise were measured at 11 though $\mathrm{t} 4$ with three items using the stem 'I intend to ...' which was followed by different levels of pelvic-floor training such as ' ... perform pelvic-floor exercise several times a week' adapted from Scholz et al. (2005).

Action planning concerning pelvic-floor exercise was measured at $\mathrm{t} 2$ and $\mathrm{t} 3$ with four items adapted from Scholz and colleagues (2005). The stem 'I have made a detailed plan regarding ...' was followed by (1) 'when'; (2) 'where'; (3) 'how'; and (4) 'how often I will perform pelvic-floor exercise'.

Since about half of the patients $(n=49 ; 43.8 \%)$ stayed at a rehabilitation clinic at $\mathrm{t} 3$ where they were instructed to perform pelvic-floor exercise, rebabilitation stay $(0=$ no, $1=$ yes) was considered as a covariate where appropriate.

The intensity of urinary incontinence was assessed at $\mathrm{t} 2, \mathrm{t} 3$, and $\mathrm{t} 4$ by the German version of the ICIQ-SF (Avery et al., 2004), with scores ranging from 0 (no incontinence) to 21 (severe incontinence). Two patients $(1.8 \%)$ faced incontinence prior surgery. At $\mathrm{t} 2,31$ patients $(27.7 \%)$ still had an indwelling catbeter $(0=$ no, $1=$ yes $)$, which was controlled for where appropriate.

Relapse from regular pelvic-floor exercise was assessed in each questionnaire. An index whether participants had interrupted pelvic-floor exercise due to health-related problems within 6 months post-surgery was used $(0=$ no, $1=$ yes $)$.

\section{Data analysis}

To account for missing data, expectation maximization was employed, including all predictors and outcomes, as well as all missing mechanisms in the imputation model (EM; NORM 2.03, Schafer, 1999). Univariate outliers were treated as suggested by Tabachnick and Fidell (2001), that is, cases with standardized scores in excess of 3.29 were reduced to one unit above the next highest value. Multivariate outliers were evaluated by means of Cook's distances. If Cook's distance exceeded 1, they were excluded from the model because of their notable influence on the model (Cook \& Weisberg, 1982). 
Hierarchical multiple regressions using SPSS 16.0 were performed to test the relative importance and unique predictive validity of phase-specific self-efficacy beliefs as competing predictors. Except for analyses involving correlates of outcomes at 6 months post-surgery ( $t 4$ ), all other analyses related predictors at $t-1$ with the outcome at $t$. We refrained from longitudinal analyses predicting outcomes at $t 4$ because of the long inter-measurement lag of 5 months. First, covariates including patients' age, educational level $(0=$ less than 12 years of schooling, $1=12$ or 13 years of schooling), nervesparing surgery $(0=$ no, $1=$ yes $)$, rehabilitation stay, incontinence or catheterization, and experience of relapses were entered in respective regression models, followed by phase-specific self-efficacy beliefs, and finally by phase-specific social-cognitive variables. For moderation analyses (Aiken \& West, 1991), predictor variables and the constituents of the respective interaction terms were centred around their grand means. In moderation models, covariates were entered first, followed by respective self-efficacy beliefs as predictor and phase-indicators as moderator, and finally by the interaction term. To display and test the interaction effects, simple slopes were tested (Preacher, Curran, \& Bauer, 2006). Low and high values of the continuous moderators were generated by adding or subtracting one standard deviation from the centred mean of the respective moderator.

\section{Results}

\section{Descriptive statistics}

The majority of the patients $(n=82 ; 73.2 \%)$ had already heard about pelvic-floor exercise, but only 10 patients ( $8.9 \%$ ) reported experience with pelvic-floor training pre-surgery. In anticipation of incontinence after surgery, participants were highly motivated to perform pelvic-floor exercise at baseline. Intention levels did not decrease until 6 months later (see Table 1). Two weeks, 1 month, and 6 months post-surgery, $27.7 \%, 10.7 \%$, and $10.7 \%$ of the patients, respectively, did not perform pelvic-floor exercise. The recommendation of at least $3 \times 10 \mathrm{~min}$ exercise per day routinely provided by physiotherapists from the Department of Urology was met by $29.5 \%(\mathrm{t} 2), 46.4 \%(\mathrm{t} 3)$, and $28.6 \%(\mathrm{t} 4)$ of the participants. Overall, 41 of the participants (36.6\%) reported that at some point between discharge and 6 months post-surgery, health-related problems had prevented them from exercising. Regarding the four phase-specific self-efficacy beliefs, patients reported medium to high levels of self-efficacies with means ranging between $2.82(S D=$ 0.78 ) and $3.67(S D=0.49)$ (see Table 1$)$. Phase-specific self-efficacies showed crosssectional intercorrelations between $r=.22$ and $r=.62$, and longitudinal correlations between $r=-.02$ and $r=.48$ (see Table 1 ). Repeated measures ANOVAs with repeated contrasts revealed that self-efficacies remained at a high level between $t 1$ and $t 2$. From $\mathrm{t} 2$ to $\mathrm{t} 4$, task self-efficacy and maintenance self-efficacy significantly decreased, whereas recovery self-efficacy did not (task self-efficacy: $F(2,110)=13.82, p<.001$; preactional self-efficacy: $F(1,111)=1.33$, n.s.; maintenance self-efficacy: $F(2,110)=12.03$, $p<.001$; recovery self-efficacy: $F(2,110)=0.91, n . s$. .

\section{Relative importance and unique predictive validity of phase-specific self-efficacy beliefs}

Testing hypothesis 1 on the relative importance and unique predictive validity of task self-efficacy as a predictor of intentions 2 weeks post-surgery, hierarchical multiple 
Table 2. Main-effect analyses on the relative importance of phase-specific self-efficacy beliefs

2A: Main-effect analysis on the relative impact of task self-efficacy predicting intentions 2 weeks post-surgery ( $\mathrm{t} 2)(\mathrm{Hypothesis} 1)$

\begin{tabular}{|c|c|c|c|c|c|c|c|c|c|}
\hline & \multicolumn{3}{|c|}{ Step I } & \multicolumn{3}{|c|}{ Step 2} & \multicolumn{3}{|c|}{ Step 3} \\
\hline & $B$ & SE B & $\beta$ & B & SE B & $\beta$ & $B$ & SE B & $\beta$ \\
\hline Age & -0.01 & 0.01 & -.07 & -0.01 & 0.01 & -.12 & -0.01 & 0.01 & -.09 \\
\hline Education & 0.08 & 0.09 & .08 & 0.14 & 0.09 & .15 & 0.11 & 0.09 & .12 \\
\hline Nerve-sparing surgery & -0.05 & 0.10 & -.05 & -0.09 & 0.09 & -.10 & -0.10 & 0.09 & -.11 \\
\hline Catheter t2 & 0.19 & 0.10 & .18 & 0.18 & 0.10 & .17 & $0.22^{*}$ & 0.10 & .21 \\
\hline Task self-efficacy $\mathrm{tl}$ & - & - & - & 0.21 & 0.11 & .22 & 0.16 & 0.12 & .16 \\
\hline Preactional self-efficacy $t \mid$ & - & - & - & 0.06 & 0.07 & .08 & 0.07 & 0.07 & .11 \\
\hline Maintenance self-efficacy $\mathrm{tl}$ & - & - & - & -0.04 & 0.07 & -.06 & -0.06 & 0.07 & -.09 \\
\hline Recovery self-efficacy tl & - & - & - & 0.14 & 0.10 & .18 & 0.13 & 0.10 & .17 \\
\hline Risk perception $\mathrm{tl}$ & - & - & - & - & - & - & -0.08 & 0.06 & -.15 \\
\hline Positive outcome-expectancies $\mathrm{tl}$ & - & - & - & - & - & - & 0.16 & 0.14 & .12 \\
\hline $\begin{array}{l}\text { Negative outcome-expectancies } \mathrm{tl} \\
\Delta R^{2}\end{array}$ & - & - & .04 & - & - & $.13^{* * *}$ & 0.09 & 0.07 & $\begin{array}{l}.13 \\
.03\end{array}$ \\
\hline
\end{tabular}


Table 2. (Continued)

2B: Main-effect analysis on the relative impact of preactional self-efficacy predicting action planning 2 weeks post-surgery ( $\mathrm{t} 2)(\mathrm{Hypothesis} 2)$

\begin{tabular}{|c|c|c|c|c|c|c|c|c|c|}
\hline & \multicolumn{3}{|c|}{ Step I } & \multicolumn{3}{|c|}{ Step 2} & \multicolumn{3}{|c|}{ Step 3} \\
\hline & B & $S E B$ & $\beta$ & B & SE B & $\beta$ & B & SE B & $\beta$ \\
\hline Age & -0.01 & 0.01 & -.04 & -0.02 & 0.01 & -.10 & -0.02 & 0.01 & -.11 \\
\hline Education & -0.15 & 0.17 & -.08 & -0.01 & 0.16 & -.01 & -0.02 & 0.16 & -.01 \\
\hline Nerve-sparing surgery & 0.20 & 0.17 & .11 & 0.15 & 0.16 & .09 & 0.18 & 0.17 & .10 \\
\hline Catheter $\mathrm{t} 2$ & $-0.38^{*}$ & 0.18 & -.20 & $-0.40^{*}$ & 0.17 & -.21 & $-0.42^{*}$ & 0.17 & -.22 \\
\hline Task self-efficacy $t \mid$ & - & - & - & 0.30 & 0.20 & .17 & 0.19 & 0.22 & .11 \\
\hline Preactional self-efficacy $\mathrm{tl}$ & - & - & - & $0.28^{*}$ & 0.12 & .24 & $0.28^{*}$ & 0.12 & .24 \\
\hline Maintenance self-efficacy tl & - & - & - & -0.01 & 0.13 & -.01 & -0.02 & 0.13 & -.02 \\
\hline Recovery self-efficacy $t I$ & - & - & - & 0.13 & 0.17 & .09 & 0.09 & 0.18 & .06 \\
\hline $\begin{array}{l}\text { Intentions tl } \\
\Delta R^{2}\end{array}$ & - & - & $-\overline{.06}$ & - & - & - & 0.27 & 0.21 & $\begin{array}{l}.14 \\
.01\end{array}$ \\
\hline
\end{tabular}


Table 2. (Continued)

$2 \mathrm{C}$ : Main-effect analysis on the relative impact of maintenance self-efficacy predicting pelvic-floor exercise $\mathrm{I}$ month post-surgery $(\mathrm{t} 3)$ (Hypothesis 3 )

\begin{tabular}{|c|c|c|c|c|c|c|c|c|c|}
\hline & \multicolumn{3}{|c|}{ Step 1} & \multicolumn{3}{|c|}{ Step 2} & \multicolumn{3}{|c|}{ Step 3} \\
\hline & $B$ & $S E B$ & $\beta$ & $B$ & SE B & $\beta$ & B & SE B & $\beta$ \\
\hline Age & -3.37 & 3.96 & -.09 & -2.94 & 3.96 & -.08 & -2.79 & 3.94 & -.07 \\
\hline Education & -27.54 & 45.33 & -.06 & -17.99 & 45.64 & -.04 & $-|4.9|$ & 45.39 & -.03 \\
\hline Nerve-sparing surgery & -48.29 & 46.09 & -.10 & -53.60 & 46.84 & -.11 & -60.44 & 46.75 & -.13 \\
\hline Rehabilitation $t 3$ & $122.99 * *$ & 45.54 & .26 & $121.59^{* *}$ & 45.37 & .26 & $119.32^{* *}$ & 45.10 & .25 \\
\hline Incontinence $t 3$ & -2.50 & 4.99 & -.05 & -2.97 & 5.06 & -.06 & -2.76 & 5.03 & -.06 \\
\hline Task self-efficacy t2 & - & - & - & -14.05 & 50.10 & -.03 & -49.54 & 54.93 & -.11 \\
\hline Preactional self-efficacy $t 2$ & - & - & - & 59.96 & 30.77 & .21 & 50.26 & 31.23 & .17 \\
\hline Maintenance self-efficacy $t 2$ & - & - & - & 9.87 & 39.89 & .03 & 18.70 & 40.05 & .05 \\
\hline Recovery self-efficacy t 2 & - & - & - & 7.13 & 38.95 & .02 & 5.45 & 38.72 & .02 \\
\hline Action planning $t 2$ & - & - & - & - & - & - & 45.40 & 29.73 & .17 \\
\hline$\Delta R^{2}$ & & & .08 & & & .05 & & & .02 \\
\hline
\end{tabular}


Table 2. (Continued)

2D: Main-effect analysis on the relative impact of recovery self-efficacy predicting pelvic-floor exercise 6 months post-surgery (t4) (Hypothesis 4)

\begin{tabular}{|c|c|c|c|c|c|c|}
\hline & \multicolumn{3}{|c|}{ Step 1} & \multicolumn{3}{|c|}{ Step 2} \\
\hline & B & $S E B$ & $\beta$ & $B$ & $S E B$ & $\beta$ \\
\hline Age & $5.08^{*}$ & 2.10 & .24 & 3.78 & 2.00 & .18 \\
\hline Education & 22.00 & 24.26 & .08 & 16.81 & 22.94 & .06 \\
\hline Nerve-sparing surgery & 28.19 & 24.57 & .11 & 22.72 & 23.81 & .09 \\
\hline Incontinence $t 4$ & $6.18^{* *}$ & 2.30 & .25 & $5.38^{*}$ & 2.17 & .22 \\
\hline Task self-efficacy t 4 & - & - & - & $75.39^{* * *}$ & 18.42 & .45 \\
\hline Maintenance self-efficacy $t 4$ & - & - & - & $-33.41^{*}$ & 16.03 & -.22 \\
\hline $\begin{array}{l}\text { Recovery self-efficacy t4 } \\
\Delta R^{2}\end{array}$ & - & - &.$\overline{.15^{* *}}$ & -8.21 & 17.95 & $\begin{array}{r}-.05 \\
.12^{* *}\end{array}$ \\
\hline
\end{tabular}

Note. ${ }^{*} p<.05 ;{ }^{* *} p<.01 ;{ }^{* * *} p<.001 . S E=$ standard error. $N=112$. 
regression analysis did not show any unique effect of any phase-specific self-efficacy. Participants who still had an indwelling catheter held higher intentions (see Table 2A).

Findings supported hypothesis 2 , in that baseline preactional self-efficacy was the strongest predictor of action planning 2 weeks post-surgery. However, participants who still had an indwelling catheter planned less (see Table 2B).

Analyses testing hypothesis 3 revealed that pelvic-floor exercise 1 month post-surgery was neither predicted by maintenance self-efficacy, nor by any other phase-specific selfefficacy belief. Attending a rehabilitation programme was related with higher pelvic-floor exercise 1 month post-surgery (see Table $2 \mathrm{C}$ ).

Due to a long time lag between $\mathrm{t} 3$ and $\mathrm{t} 4$ of 5 months, we chose to perform cross-sectional analyses predicting pelvic-floor exercise 6 months post-surgery for a test of hypothesis 4 . At this time, task self-efficacy was positively and maintenance self-efficacy was negatively associated with pelvic-floor exercise. No main effect for recovery self-efficacy was found. Additionally, pelvic-floor exercise was strongly related to incontinence 6 months post-surgery (see Table 2D).

\section{Phase-specificity of self-efficacy beliefs: Exploratory moderation analyses}

Exploratory analyses tested differential relations between phase-specific self-efficacy and respective outcomes moderated by indicators of phase-allocation. Predicting action planning 2 days post-surgery, preactional self-efficacy and intentions did not interact. Instead, action planning was positively associated with intentions and negatively associated with catheterization (see Table 3A). One multivariate outlier had to be removed from analyses due to high influence on the model.

Considering pelvic-floor exercise at 2 weeks post-surgery as an indicator of allocation to the maintenance phase, neither a moderation effect, nor a main effect of maintenance self-efficacy were found. Instead, past behaviour was the best predictor of pelvic-floor exercise 1 month post-surgery (see Table 3B).

At 6 months post-surgery, relapses moderated effects of recovery self-efficacy on pelvic-floor training (see Table 3C). Simple slope analyses showed that recovery selfefficacy was associated with returns to pelvic-floor after relapse $(B=74.06, p<.05)$, whereas there was no such association in participants without relapses $(B=-15.41$, n.s.). Incontinence and age were positively related to pelvic-floor exercise 6 months post-surgery.

\section{Discussion}

Based on phase assumptions of the HAPA model, the present study tested the theoretical differentiation between and relative importance of four phase-specific self-efficacy beliefs in health-behaviour change.

Contrary to predictions and prior findings (Scholz et al., 2005), task self-efficacy immediately following surgery was not uniquely associated with intentional strength 2 weeks later. Next to a fairly high overlap with other phase-specific self-efficacy beliefs, notably, recovery self-efficacy, the lack of unique associations in this analysis may also be due to unstable predictors (Ajzen, 1996) or overstated ratings at baseline. Initially, high levels of motivational predictors and high intentions shortly following the operation might have been adjusted following discharge to be more compatible with patients' everyday life (Sniehotta, Scholz, et al., 2005). 
Table 3. Exploratory moderation analyses on the phase-specificity of self-efficacy beliefs

3A: Moderation analysis on preactional self-efficacy by level of intention predicting action planning 2 weeks post-surgery $(\mathrm{t} 2)(n=11 \mathrm{I})$

\begin{tabular}{|c|c|c|c|c|c|c|}
\hline & \multicolumn{3}{|c|}{ Step I } & \multicolumn{3}{|c|}{ Step 2} \\
\hline & B & $S E B$ & $\beta$ & B & SE B & $\beta$ \\
\hline Age & -0.02 & 0.01 & -.11 & -0.02 & 0.01 & -.14 \\
\hline Education & -0.23 & 0.16 & -.13 & -0.10 & 0.16 & -.06 \\
\hline Nerve-sparing surgery & 0.24 & 0.16 & .14 & 0.25 & 0.16 & .15 \\
\hline Catheter $\mathrm{t} 2$ & $-0.43^{*}$ & 0.17 & -.23 & $-0.43^{*}$ & 0.17 & -.23 \\
\hline Preactional self-efficacy $t \mid(A)$ & - & - & - & 0.20 & 0.12 & .17 \\
\hline Intentions tl (B) & - & - & - & $0.45^{*}$ & 0.19 & .23 \\
\hline$A \times B$ & - & - & - & 0.60 & 0.35 & .18 \\
\hline$\Delta R^{2}$ & & & $.09 *$ & & & $.10^{* *}$ \\
\hline
\end{tabular}


Table 3. (Continued)

3B: Moderation analysis on maintenance self-efficacy by level of past behaviour predicting pelvic-floor exercise I month post-surgery ( $\mathrm{t} 3$ )

\begin{tabular}{|c|c|c|c|c|c|c|}
\hline & \multicolumn{3}{|c|}{ Step 1} & \multicolumn{3}{|c|}{ Step 2} \\
\hline & $B$ & $S E B$ & $\beta$ & B & $S E B$ & $\beta$ \\
\hline Age & -3.37 & 3.96 & -.09 & -2.84 & 3.80 & -.07 \\
\hline Education & -27.54 & 45.33 & -.06 & -8.83 & 43.77 & -.02 \\
\hline Nerve-sparing surgery & -48.29 & 46.09 & -.10 & -60.57 & 44.07 & -.13 \\
\hline Rehabilitation $\mathrm{t} 3$ & $122.99^{* *}$ & 45.54 & .26 & $108.11^{*}$ & 43.74 & .23 \\
\hline Incontinence $t 3$ & -2.51 & 5.00 & -.05 & -0.57 & 4.96 & -.01 \\
\hline Maintenance self-efficacy t2 (A) & - & - & - & 37.67 & 33.85 & .10 \\
\hline Pelvic-floor exercise t2 (B) & - & - & - & $0.45^{* *}$ & 0.13 & .31 \\
\hline$A \times B$ & - & - & - & 0.10 & 0.20 & .05 \\
\hline$\Delta R^{2}$ & & & .08 & & & $.11 * *$ \\
\hline
\end{tabular}


Table 3. (Continued)

3C: Moderation analysis on recovery self-efficacy by occurrence of relapse predicting pelvic-floor exercise 6 months post-surgery ( $(4)$

\begin{tabular}{|c|c|c|c|c|c|c|}
\hline & \multicolumn{3}{|c|}{ Step I } & \multicolumn{3}{|c|}{ Step 2} \\
\hline & B & $S E B$ & $\beta$ & B & $S E B$ & $\beta$ \\
\hline Age & $5.08^{*}$ & 2.10 & .24 & $4.31^{*}$ & 2.09 & .20 \\
\hline Education & 22.00 & 24.26 & .08 & 20.61 & 23.99 & .08 \\
\hline Nerve-sparing surgery & 28.19 & 24.57 & .11 & 22.62 & 24.89 & .09 \\
\hline Incontinence $\mathrm{t} 4$ & $6.18^{* *}$ & 2.30 & .25 & $6.14^{* *}$ & 2.28 & .25 \\
\hline Recovery self-efficacy t 4 (A) & - & - & - & 29.33 & 17.56 & .17 \\
\hline Lapses (B) & - & - & - & -9.03 & 23.97 & -.03 \\
\hline$A \times B$ & - & - & - & $93.20^{*}$ & 39.63 & .23 \\
\hline$\Delta R^{2}$ & & & $.15^{* *}$ & & & .05 \\
\hline
\end{tabular}

Note. ${ }^{*} p<.05 ;{ }^{* *} p<.01 . S E=$ standard error. $N=112$. 
In the preactional phase, behaviour initiation needs to be planned. Extending previous findings (e.g., Luszczynska \& Schwarzer, 2003), our results suggest preactional selfefficacy to be a better predictor for planning than task self-efficacy or any other of the tested phase-specific self-efficacy beliefs. Specifically, the differentiation between task and preactional self-efficacy indicates that different steps towards action (i.e., intention formation and planning), are supported by distinct self-efficacy beliefs. One could argue that a high similarity of predictors and outcomes in terms of context, content, generalizability, and/or salient beliefs (Ajzen, 1996) explain this pattern of effects. In the present study, however, predictors and outcomes differed in terms of measurement context, that is, before and after discharge from the hospital. Moreover, preactional self-efficacy was operationalized as confidence in one's preparatory actions, whereas planning is but one aspect of action preparation that also includes learning and selfregulation. Thus, in endorsing preactional self-efficacy items, diverse beliefs are activated which go beyond beliefs associated with action-planning.

Considering intention strength as a moderator did not further qualify this main effect of preactional self-efficacy on action planning. A number of explanations might account for this. As the present sample was fairly small, power problems are a likely explanation for our failure to detect an interaction effect (McClelland \& Judd, 1993). Moreover, patients' intentions to perform regular pelvic-floor exercise were almost uniformly high, making detection of a differential prediction difficult.

The assessment 1 month post-surgery was chosen to represent the maintenance phase, assuming that patients have started to exercise their pelvic-floor regularly at this point. In contrast to our hypotheses and findings from previous longitudinal studies (e.g., Luszczynska \& Schwarzer, 2003; Schwarzer \& Renner, 2000), maintenance self-efficacy 2 weeks post-surgery was neither directly associated with pelvic-floor exercise 1 month post-surgery, nor moderated by past behaviour. As many patients were still catheterized at 2 weeks post-surgery, they might not have had sufficient opportunity to gain maintenance self-efficacy beliefs by mastery experiences (Bandura, 1977). Additionally, participants with long catheterization times might not yet have reached the maintenance phase 1 month post-surgery. Also, context effects might have overruled predictive power of maintenance self-efficacy at this point. Analyses indicated that attending rehabilitation 1 month post-surgery was the strongest predictor of pelvic-floor exercise at that time. Within a structured environment, that is, in the context of a rehabilitation programme, maintenance self-efficacy might lose importance as a predictor of pelvic-floor exercise that is a scheduled part of the programme. The present study thus failed to confirm phase-specific effects of maintenance self-efficacy in persons who already acted.

Investigating the relative importance of phase-specific self-efficacy beliefs with regard to pelvic-floor exercise 6 months post-surgery, unexpectedly, task self-efficacy rather than recovery self-efficacy turned out to be the strongest predictor. One explanation for the missing main effect of recovery self-efficacy might be that $63 \%$ of the participants did not report relapses and therefore did not need or build up recovery self-efficacy. We thus also explored the moderating role of prior relapse in the association between recovery self-efficacy and pelvic-floor exercise. In line with previous findings (Scholz et al., 2005), we found that only participants experiencing such relapses benefitted from recovery self-efficacy beliefs in terms of pelvic-floor exercise.

In case of the unexpected main effect of task self-efficacy in the maintenance of pelvic-floor exercise 6 months post-surgery, level of measurement might be addressed as an explanation. In that, task self-efficacy, operationalized as an overall confidence in changing the respective behaviour might reflect a higher order, more conceptional, and 
less specific measurement approach when compared to other types of phase-specific self-efficacy beliefs tested in this study. Although four types of self-efficacy beliefs were distinguished by means of exploratory principal components analysis with high internal consistencies of the four subscales, indicating satisfying construct validity in the present study, future research should address this issue in more detail.

In contrast to assumptions derived from the HAPA model, maintenance self-efficacy turned out to be negatively associated with pelvic-floor exercise 6 months post-surgery. This conflicts with findings from studies with comparable time frames (Luszczynska \& Schwarzer, 2003; Schwarzer \& Renner, 2000). Supporting the HAPA assumptions, these studies investigated phase-specific self-efficacy in the context of physical exercise in university students (Rodgers et al., 2002), in patients after myocardial infarction (Scholz et al., 2005), or in women performing breast self-examination (Luszczynska, 2004) that is, health behaviours that have to be maintained for longer time periods to be efficient. Study context may therefore explain why positive associations between maintenance self-efficacy and behaviour were found in these studies, but not in the present study. The need to perform pelvic-floor exercise depends on whether patients still experience incontinence symptoms. As our results show, only participants with stronger incontinence continued to exercise on a high level, whereas those with lower incontinence symptoms exercised less. Thus, participants who already gained maintenance self-efficacy beliefs 1 month post-surgery were able to sustain and then reduce their exercise earlier. Alternatively, the negative association between maintenance selfefficacy and pelvic-floor exercise 6 months post-surgery may be explained by suppressor effects due to strong predictors such as task self-efficacy in the model (Shrout $\&$ Bolger, 2002). This result may, however, also raise the theoretical question whether behaviours such as pelvic-floor exercise, which are performed several times a day, still need to be actively 'maintained' or whether they are already habituated and thus do not require maintenance self-efficacy anymore. Future research might focus on processes such as, habituation and disengagement.

Regarding prior evidence of increasing self-efficacy as behaviour change progresses (Sniehotta, Luszczynska, Scholz, \& Lippke, 2005), we also found changes, but decreases in task and maintenance self-efficacy beliefs from 1 month to 6 months post-surgery. These led to low longitudinal intercorrelations of self-efficacy beliefs. This might reflect habituation of self-efficacy beliefs throughout the behaviour change progress. Participants might not need to recall them consciously. Moreover, these changes and different predictive patterns of phase-specific self-efficacy beliefs underscore the importance of differentiating between phase-specific confidences in one's capabilities to cope with different challenges during the preparation, implementation, and maintenance of a new health behaviour.

In addition to limitations already discussed, the present findings might be limited by a number of other factors. Behavioural outcomes were assessed by self-reports only, using widely spaced inter-measurement intervals (Schwarz, 1999). While the former may call into question the validity of the assessment of the primary outcome, the latter prevented us from taking a closer look at processes of behaviour change. Future work should back up self-reports by objective measures where applicable and use diaries or weekly measurement to capture processes of change. Moreover, the operationalization of phases and the identification of indicators of phase allocation turned out to be very important in this longitudinal design. Future research should investigate additional measurement points following the initiation of behaviour in order to capture stage transitions within short time intervals. Finally, using larger samples, also including female and mixed 
samples, and investigating different health behaviours, future research should replicate and test generalizability of the present findings.

Concluding, this study investigated phase-specific self-efficacy in the context of uptake and maintenance of pelvic-floor exercise following radical prostatectomy. Although theory assumes specific self-efficacy beliefs for each phase of health-behaviour change (Schwarzer, 2001), the present study failed to fully support these theoretical assumptions. Findings nevertheless supported differentiation between preintentional (i.e., task self-efficacy) and a preactional self-efficacy during early phases of behaviour change. Moreover, the present results stress the importance of considering the occurrence of relapses as a moderator of potential effects of recovery self-efficacy on the maintenance of behaviour change. However, inconsistent findings also draw attention to the need for precise operational definitions of phase-specific self-efficacy beliefs and continued investigation, including experimental manipulation or quasi-experimental work using matched-mismatched designs.

More knowledge on the role of phase-specific self-efficacy beliefs in health-behaviour change might inform further development of tailored interventions. Added intervention strategies could strengthen individuals' specific confidence in their ability to overcome challenges along the way.

\section{References}

Abraham, C. (2008). Beyond stages of change: Multi-determinant continuum models of action readiness and menu-based interventions. Applied Psycbology: An International Review, 57, 30-41. doi:10.1111/j.1464-0597.2007.00320.x

Aiken, L. S., \& West, S. G. (1991). Multiple regression: Testing and interpreting interactions. Newbury Park, CA: Sage.

Ajzen, I. (1996). The direct influence of attitudes on behavior. In P. M. Gollwitzer (Ed.), The psychology of action (pp. 385-403). New York: Guilford.

Avery, K., Donovan, J., Peters, T. J., Shaw, C., Gotoh, M., \& Abrams, P. (2004). ICIQ: A brief and robust measure for evaluating the symptoms and impact of urinary incontinence. Neurourology and Urodynamics, 23, 322-330. doi:10.1002/nau.20041

Bandura, A. (1977). Self-efficacy: Toward a unifying theory of behavioral change. Psychological Review, 84, 191-215. doi:10.1037/0033-295X.84.2.191

Bandura, A. (1997). Self-efficacy: The exercise of control. New York: Freeman. doi:10.1002/ 9780470479216.corpsy0836

Basillote, J. B., Ahlering, T. E., Skarecky, D. W., Lee, D. I., \& Clayman, R. V. (2004). Laparoscopic radical prostatectomy. Review and assessment of an emerging technique. Surgical Endoscopy, 18, 1694-1711. doi:10.1007/s00464-003-8267-x

Chow, S., \& Mullan, B. (2010). Predicting food hygiene: An investigation of social factors and past behaviour in an extended model of the Health Action Process Approach. Appetite, 54, 126-133. doi:10.1016/j.appet.2009.09.018

Cook, R. D., \& Weisberg, S. (1982). Residuals and influence in regression. New York: Chapman \& Hall. doi:10.1002/bimj.4710270110

Debruyne, F. M. J., \& Beerlage, H. P. (2000). The place of radical prostatectomy in the treatment of early localized prostate cancer. Radiotherapy and Oncology, 57, 259-262. doi:10.1016/ S0167-8140(00)00285-1

Dorey, G., Glazener, C., Buckley, B., Cochran, C., \& Moore, K. (2009). Developing a pelvicfloor muscle training regimen for use in a trial intervention. Physiotherapy, 95, 99-128. doi:10.1016/j.physio. 2009.03.003

Ferlay, J., Bray, F., Pisani, P., \& Parkin, D. M. (2001). GLOBOCAN 2000: Cancer Incidence, Mortality, and Prevalence Worldwide, Version 1.0, IARC CancerBase No. 5. Lyon, France: International Agency for Research on Cancer and World Health Organization, IARC Press. 
Gollwitzer, P. M. (1999). Implementation intentions: Strong effects of simple plans. American Psychologist, 54, 493-503. doi:10.1037/0003-066X.54.7.493

Gollwitzer, P. M., \& Sheeran, P. (2006). Implementation intentions and goal achievement: A metaanalysis of effects and processes. Advances in Experimental Social Psychology, 38, 249-268. doi:10.1016/S0065-2601(06)38002-1

Hunter, K. F., Moore, K. N., \& Glazener, C. M. A. (2007). Conservative management for prostatectomy urinary incontinence. Cochrane Database of Systematic Reviews, 2, No.: CD001843. doi: 10.1002/14651858.CD001843.pub3

Leventhal, H., Singer, R., \& Jones, S. (1965). Effects of fear and specificity of recommendation upon attitudes and behaviour. Journal of Personality and Social Psychology, 34, 20-29. doi: $10.1037 / \mathrm{h} 0022089$

Lippke, S., Ziegelmann, J. P., \& Schwarzer, R. (2005). Stage-specific adoption and maintenance of physical activity: Testing a three-stage model. Psycbology of Sport and Exercise, 6, 585-603. doi:10.1016/j.psychsport.2004.11.002

Luszczynska, A. (2004). Change in breast self-examination behavior: Effects of intervention on enhancing self-efficacy. Internatinal Journal of Bebavioral Medicine, 11, 95-103. doi:10. 1207/s15327558ijbm1102_5

Luszczynska, A., \& Schwarzer, R. (2003). Planning and self-efficacy in the adoption and maintenance of breast self-examination: A longitudinal study on self-regulatory cognitions. Psychology and Health, 18, 93-108. doi:10.1080/0887044021000019358

Marlatt, G. A., Baer, J. S., \& Quigley, L.A. (1995). Self-efficacy and addictive behavior. In A. Bandura (Ed.), Self-efficacy in changing societies (pp. 289-315). New York: Cambridge University Press. doi: $10.2277 / 0521586968$

McClelland, G. H. \& Judd, C. M. (1993). Statistical difficulties of detecting interactions and moderator effects. Psychological Bulletin, 114, 376-390. doi:10.1037/0033-2909.114.2.376

Preacher, K. J., Curran, P. J., \& Bauer, D. J. (2006). Computational tools for probing interaction effects in multiple linear regression, multilevel modeling, and latent curve analysis. Journal of Educational and Behavioral Statistics, 31, 437-448. doi:10.3102/10769986031004437

Renner, B., Spivak, Y., Kwon, S., \& Schwarzer, R. (2007). Does age make a difference? Predicting physical activity of South Koreans. Psychology and Aging, 22, 482-493. doi:10.1037/ 0882-7974.22.3.482

Rodgers, W. M., Hall, C. R., Blanchard, C. M., McAuley, E., \& Munroe, K. J. (2002). Task and scheduling self-efficacy as predictors of exercise behavior. Psychology \& Health, 17, 405-416. doi: $10.1080 / 0887044022000004902$

Schafer, J. L. (1999). NORM: Multiple imputation of incomplete multivariate data under a normal model, version 2.03 [Computer software]. Retrieved from: http://www.stat.psu.edu/ $\sim$ jls/misoftwa.html

Scholz, U., Sniehotta, F. F., \& Schwarzer, R. (2005). Predicting physical exercise in cardiac rehabilitation: The role of phase-specific self-efficacy beliefs. Journal of Sport and Exercise Psychology, 27, 135-151.

Schwarz, N. (1999). Self-reports: How the question shapes the answer. American Psychologist, 54,93-105. doi:10.1037/0003-066X.54.2.93

Schwarzer, R. (2001). Social-cognitive factors in changing health-related behaviors. Current Directions in Psychological Science, 10, 47-51. doi:10.1111/1467-8721.00112

Schwarzer, R. (2008). Modeling health behaviour change: How to predict and modify the adoption and maintenance of health behaviors. Applied Psychology: An International Review, 57, 1-29. doi:10.1111/apps.2008.57.issue-1

Schwarzer, R., \& Renner, B. (2000). Social-cognitive predictors of health behavior: Action selfefficacy and coping self-efficacy. Health Psychology, 5, 487-495. doi:10.1037/0278-6133.19. 5.487

Schwarzer, R., Schüz, B., Ziegelmann, J. P., Lippke, S., Luszczynska, A., \& Scholz, U. (2007). Adoption and maintenance of four health behaviors: Theory-guided longitudinal studies on 
dental flossing, seat belt use, dietary behavior, and physical activity. Annals of Bebavioral Medicine, 33, 156-166. doi:10.1007/BF02879897

Shrout, P. E., \& Bolger, N. (2002). Mediation in experimental and nonexperimental studies: New procedures and recommendations. Psychological Methods, 7, 422-445. doi:10.1037/ 1082-989X.7.4.422

Sniehotta, F. F., Luszczynska, A., Scholz, U., \& Lippke, S. (2005). Discontinuity patterns in stages of the precaution adoption process model: Meat consumption during a livestock epidemic. British Journal of Health Psychology, 10, 221-235. doi:10.1348/135910705X26137

Sniehotta, F. F., Scholz, U., Schwarzer, R., Fuhrmann, B., Kiwus, U., \& Völler, H. (2005). Long-term effects of two psychological interventions on physical exercise and self-regulation following coronary rehabilitation. International Journal of Behavioral Medicine, 12, 244-255. doi:10. 1207/s15327558ijbm1204_5

Tabachnick, B. G., \& Fidell, L. S. (2001). Using multivariate statistics (4th ed.). Boston, MA: Allyn \& Bacon.

Wiedemann, A. U., Lippke, S., Reuter, T., Schüz, B., Ziegelmann, J. P., \& Schwarzer, R. (2009). Prediction of stage transition in fruit and vegetable intake. Health Education Research, 24, 596-607. doi:10.1093/her/cym092 